\title{
The hidden symmetry-breaking picture of symmetry-protected topological order
}

\author{
Dominic V. Else, ${ }^{1,2}$ Stephen D. Bartlett, ${ }^{1}$ and Andrew C. Doherty ${ }^{1}$ \\ ${ }^{1}$ Centre for Engineered Quantum Systems, School of Physics, \\ The University of Sydney, Sydney, NSW 2006, Australia \\ ${ }^{2}$ Department of Physics, University of California, Santa Barbara, CA 93106, USA
}

\begin{abstract}
We generalize the hidden symmetry-breaking picture of symmetry-protected topological (SPT) order developed by Kennedy and Tasaki in the context of the Haldane phase. Our generalization applies to a wide class of SPT phases in one-dimensional spin chains, protected by an on-site representation of a finite abelian group. This generalization takes the form of a non-local unitary map that relates local symmetry-respecting Hamiltonians in an SPT phase to local Hamiltonians in a symmetry-broken phase. Using this unitary, we establish a relation between the two-point correlation functions that characterize fully symmetry-broken phases with the string-order correlation functions that characterise the SPT phases, therefore establishing the perspective in these systems that SPT phases are characterised by hidden symmetry-breaking. Our generalization is also applied to systems with continuous symmetries, including $\mathrm{SO}(2 k+1)$ and $\mathrm{SU}(k)$.
\end{abstract}

PACS numbers: $64.70 . \mathrm{Tg}, 75.10 . \mathrm{Pq}$

\section{INTRODUCTION}

In the traditional Landau paradigm, order in condensed matter systems is viewed as synonymous with the spontaneous breaking of a symmetry. However, it is now well-established that at zero temperature there exist topologically ordered phases, such as those of the fractional quantum Hall effect, which cannot be understood through the Landau paradigm. Additionally, one can also consider symmetry-protected topological (SPT) phases ${ }^{1}$, which are not topologically ordered in the conventional sense, yet remain distinct from the trivial phase in the presence of an appropriate symmetry. A wellknown example of an SPT phase is the Haldane phase of antiferromagnetic spin- 1 chains, which is protected by the $D_{2} \cong Z_{2} \times Z_{2}$ symmetry comprising $\pi$ rotations about a set of orthogonal axes. The "topological" nature of the Haldane phase is manifested in a number of ways, such as the long-range string order ${ }^{2}$, fractionalized edge modes ${ }^{3}$, degenerate entanglement spectrum ${ }^{4}$, and long-range localizable entanglement ${ }^{516}$. It is now known that many of the interesting properties of the Haldane phase extend in general to SPT phases of one-dimensional spin chains protected by a unitary "on-site" representation of an arbitrary symmetry group $G$ (for which the Haldane phase, with $G=Z_{2} \times Z_{2}$, is the simplest non-trivial example), which have been fully classified 78 .

An early and influential characterization of the Haldane phase was provided by Kennedy and Tasak ${ }^{9}$ (see also Ref. (10). They constructed a non-local unitary [which we refer to as the Kennedy-Tasaki (KT) transformation] to transform the Haldane phase to a conventional symmetry-breaking phase. Although the transformation is non-local, for any local Hamiltonian $H$ that respects the $D_{2}$ rotation symmetry, the KT transformation yields another $D_{2}$-symmetric local Hamiltonian $\widetilde{H}$. It turns out that if $H$ is in the SPT Haldane phase, then $\widetilde{H}$ will have a space of four degenerate locally-distinguishable ground states corresponding to the spontaneous breaking of the $D_{2}$ rotation symmetry, i.e. $\widetilde{H}$ is in the maximal symmetry-breaking phase for the $D_{2}$ symmetry. Thus, the ordering in the SPT phase is interpreted as "hidden symmetry-breaking". Furthermore, the long-ranged string order in the Haldane phase is related by the KT transformation to conventional long-ranged order in the symmetry breaking phase.

The Haldane phase is also a special case of the SPT phases that were classified in Refs. 7 and 8 through the symmetry properties of a matrix-product state ansatz for the ground state. The general result is that the distinct SPT phases with respect to an on-site representation of a symmetry group $G$ are classified by the second cohomology classes of the projective representations of the symmetry group. This classification appears to be the most fundamental and general approach to SPT phases for one-dimensional chains with an on-site symmetry. However, the connection with the original hidden symmetrybreaking picture of Kennedy and Tasaki has not been explored. As a result, it remains unclear to what extent other SPT phases can also be understood to arise from a similar hidden symmetry-breaking mechanism (although see Ref. 11 for one example).

In this paper, we extend the hidden-symmetry breaking picture to any SPT phase protected in a onedimensional spin chain by an on-site representation of a finite abelian group, provided that the cohomology class describing the phase satisfies a condition called maximal non-commutativity. (We say that a cohomology class is maximally non-commutative if, in the corresponding projective representations, for the matrix representation of any non-trivial group element there exists at least one other matrix in the representation with which it does not commute ${ }^{12}$.) We achieve this by constructing a suitable generalization of the KT transformation (presented in Sec. IV], expressed explicitly in terms of the appropriate cohomology class of the symmetry group, to transform the SPT phase into a conventional symmetry-breaking 
phase. The generalized KT transformation is essentially equivalent to the duality transformation introduced by us in the context of quantum computation 13 , and some of its properties were already discussed in the appendices of that paper; however, our treatment here will be selfcontained.

Where it can be applied, our generalized KT transformation affords a different perspective on properties of the SPT phase. For example, for abelian symmetry groups, SPT phases (and indeed, all symmetric phases) can be identified from a pattern of string order (as we will show, based in part on the results of Ref. 14). For an SPT phase corresponding to a maximally non-commutative cohomology class, this pattern of string order can be understood in a natural way through the generalized KT transformation, which relates it to the long-range order characterizing the symmetry-breaking phase (just as the original KT transformation does in the case of the Haldane phase). We explore this perspective in Sec. VI. We remark that, although we are only able to consider finite abelian symmetry groups, these groups can arise as subgroups for systems with a larger symmetry. For SPT phases in systems that have a $\mathrm{SO}(2 k+1)$ or $\mathrm{SU}(k)$ symmetry, in Sec. VII we will exhibit an appropriate finite abelian subgroup that allows the hidden symmetry breaking to be identified.

We note that a closely related investigation, Ref. 15, appeared shortly after our work and contains similar results to ours. Where relevant, we will remark on some of the similarities and differences between the two works. In particular, our generalized KT transformation coincides with that of Ref. 15 for the specific case of a $Z_{N} \times Z_{N}$ symmetry group.

\section{THE KENNEDY-TASAKI TRANSFORMATION}

Let us recall the definition of the unitary $\mathcal{D}_{K T}$ that effects the Kennedy-Tasaki transformation for a chain of $N$ spin-1's with open boundary conditions. It can be written as $\$ 16$

$$
\mathcal{D}_{K T}=\prod_{j<k} \exp \left(i \pi S_{j}^{z} S_{k}^{x}\right)
$$

where $S_{j}^{a}(a=x, y, z)$ denotes the appropriate spin component operator for the $j$-th spin. This unitary is nonlocal, but, for any local observable $A$ that respects the $D_{2}$ symmetry operations $\prod_{j} \exp \left(i \pi S_{j}^{a}\right)(a=x, y, z)$, the transformed observable $\mathcal{D}_{K T} A \mathcal{D}_{K T}^{\dagger}$ remains local and symmetry-respecting. Therefore, for any Hamiltonian $H$ that is the sum of local symmetry-respecting interactions, one can generate the dual Hamiltonian $\widetilde{H}=\mathcal{D}_{K T} H \mathcal{D}_{K T}^{\dagger}$. If $H$ is in the SPT phase with respect to the $D_{2}$ symmetry, then $\widetilde{H}$ is expected to be in a conventional symmetrybreaking phase with respect to the symmetry, with the four-fold degenerate edge states mapping under $\mathcal{D}_{K T}$ to the four locally distinguishable symmetry-breaking ground states.

In order to see that $\mathcal{D}_{K T}$ is a special case of the generalized KT transformation to be defined later, we will want to express $\mathcal{D}_{K T}$ in terms of the single-site basis $\{|x\rangle,|y\rangle,|z\rangle\}$ (where $|a\rangle$ is the zero eigenstate of $S^{a}$ for $a=x, y, z)$, that is the simultaneous eigenbasis of the on-site representation of the symmetry. Observe that

$$
\exp \left(i \pi S_{z} \otimes S_{x}\right)\left(\left|a_{1}\right\rangle \otimes\left|a_{2}\right\rangle\right)=(-1)^{\mu\left(a_{1}\right) \nu\left(a_{2}\right)}\left|a_{1}\right\rangle \otimes\left|a_{2}\right\rangle,
$$

where $\mu(a)=1-\delta_{a, z}$ and $\nu(a)=1-\delta_{a, x}$. Hence we can write

$$
\mathcal{D}_{K T}=\sum_{a_{1}, \ldots, a_{N}}(-1)^{\sum_{j<k} \mu\left(a_{j}\right) \nu\left(a_{k}\right)}\left|a_{1}, \ldots, a_{N}\right\rangle\left\langle a_{1}, \ldots, a_{N}\right| .
$$

\section{CLASSIFICATION OF SPT PHASES BY COHOMOLOGY CLASSES}

Throughout this paper, we will assume a chain of $N$ spins, such that the Hamiltonian commutes with the on-site representation $[u(g)]^{\otimes N}$ of a symmetry group $G$. We will assume open boundary conditions unless otherwise stated. According to the general classification of SPT phases for on-site symmetries in one-dimensional systems ${ }^{78}$, the different SPT phases for this symmetry can be classified by the second cohomology group $H^{2}(G, \mathrm{U}(1))$, which is related to the projective representations $V(g)$ for the group $G$, as we now describe. (One interpretation of these projective representations is that they describe the action of the symmetry on the fractionalized edge mode associated with each edge for open boundary conditions; we note that there are several subtleties with the formal treatment of such edge modes, and we refer the reader to Sec. 3.1 of Ref. 13 for a careful discussion.) By definition, a projective representation $V(g)$ must satisfy

$$
V\left(g_{1}\right) V\left(g_{2}\right)=\omega\left(g_{1}, g_{2}\right) V\left(g_{1} g_{2}\right), \quad \forall g_{1}, g_{2} \in G,
$$

where $\omega$ is a function mapping pairs of group elements to complex phase factors, known as the factor system of the projective representation. The associativity of matrix multiplication implies that the factor system must satisfy the 2-cocycle condition

$$
\begin{array}{r}
\omega\left(g_{1}, g_{2}\right) \omega\left(g_{1} g_{2}, g_{3}\right)=\omega\left(g_{2}, g_{3}\right) \omega\left(g_{1}, g_{2} g_{3}\right), \\
\forall g_{1}, g_{2}, g_{3} \in G .
\end{array}
$$

Conversely, any $\omega$ satisfying Eq. (5) is the factor system for some projective representation 17 . Furthermore, given any projective representation, it is trivial to generate another one by rephasing of the operators $V(g)$, i.e. $V(g) \rightarrow \beta(g) V(g)$, where $\beta$ is a function that sends 
group elements to phase factors. The effect on the factor system is

$$
\omega\left(g_{1}, g_{2}\right) \rightarrow \beta\left(g_{1} g_{2}\right)^{-1} \beta\left(g_{1}\right) \beta\left(g_{2}\right) \omega\left(g_{1}, g_{2}\right) .
$$

Two factor systems related by a transformation of the form Eq. (6) are said to be in the same cohomology class, and the second cohomology group $H^{2}(G, \mathrm{U}(1))$ comprises all the distinct cohomology classes for the group $G$. We will denote by $[\omega]$ the cohomology class containing a given factor system $\omega$.

In the case of the Haldane phase for spin- 1 chains, the relevant symmetry group is $D_{2}=\{1, x, y, z\}$ (where $y=$ $x z)$, with the on-site representation $u(a)=\exp \left(i \pi S_{a}\right)$ (for $a=x, y, z$ ). The Haldane phase corresponds to the unique non-trivial cohomology class for the symmetry group $D_{2}$. We can specify a representative factor system $\omega$ for this cohomology class by giving an example of a projective representation for which $\omega$ is the factor system, namely

$$
V(1)=\mathbb{I}, \quad V(x)=\sigma_{x}, \quad V(z)=\sigma_{z}, \quad V(y)=\sigma_{x} \sigma_{z},
$$

where $\sigma_{x}$ and $\sigma_{z}$ are the respective Pauli spin matrices. We define $V(y)$ as above, rather than the more symmetrical $V(y)=\sigma_{y}$ (which would correspond to a different factor system within the same cohomology class), because the factor system of Eq. (7) will turn out to be closely connected to the conventional formulation of the KT transformation.

\section{THE GENERALIZED KENNEDY-TASAKI TRANSFORMATION}

In this section, we will define our generalized KT transformation, for an SPT phase characterized by a cohomology class $[\omega]$ and an on-site symmetry representation of a group $G$. We will require that $G$ be finite and abelian, and that the cohomology class $[\omega]$ be maximally non-commutative (to be defined below). A special property of an abelian symmetry is that the irreps are one-dimensional; therefore, the on-site representation $u(g)$ must decompose as

$$
u(g)=\bigoplus_{\chi} \chi(g) \mathbb{I}_{m_{\chi}}
$$

where the sum is over the one-dimensional representations (characters) $\chi$ of $G$. For simplicity of presentation, we assume that none of the multiplicities $m_{\chi}$ are greater than 1 ; thus, we can write

$$
u(g)=\sum_{\chi} \chi(g)|\chi\rangle\langle\chi|
$$

where the $\{|\chi\rangle\}$ form an orthonormal basis, and the sum is over those $\chi$ such that $m_{\chi}>0$. However, all the results of this paper can easily be generalized to the case of multiplicities greater than 1. For the Haldane phase, this basis $\{|\chi\rangle\}$ is the basis $\{|x\rangle,|y\rangle,|z\rangle\}$ discussed in Sec. II

Our generalized construction applies for any SPT phase with respect to the aforementioned symmetry, so long as the corresponding cohomology class $[\omega]$ is maximally-noncommutative, which is to say that the subgroup $G(\omega)=\left\{g \in G: V_{\omega}(g) V_{\omega}(h)=V_{\omega}(h) V_{\omega}(g) \forall h \in\right.$ $G\}$ is trivial. This property does not depend on the choice of representative factor system for the cohomology class. (Throughout this section, we will use $V_{\omega}$ to denote some projective representation of $G$ with factor system $\omega$; it does not matter how the projective representation is chosen because we only use the multiplicative relations between the matrices $V_{\omega}(g)$, and these are determined by $\omega$.$) As follows from Refs. 17 and 18, a finite abelian group$ $G$ will have at least one maximally non-commutative factor system if and only if it is of "symmetric type", i.e. $G \cong H \times H$ for some group $H$. Of course, even if the full symmetry group is not of this form, then it might still have a subgroup of symmetric type, for which our method could be applied.

An important property of a maximally noncommutative factor system is the following. Any cohomology class for an abelian group can be considered to induce a homomorphism $\varphi_{\omega}$ from $G$ to $G^{*}$ (where $G^{*}$ is the character group of $G$, i.e. the group of one-dimensional projective representations of $G$ under multiplication), according to

$$
\varphi_{\omega}(g)=\chi_{g}^{\omega},
$$

where $\chi_{g}^{\omega}$ is the one-dimensional representation of $G$ such that

$$
V_{\omega}\left(g^{\prime}\right) V_{\omega}(g) V_{\omega}\left(g^{\prime}\right)^{\dagger}=\chi_{g}^{\omega}\left(g^{\prime}\right) V_{\omega}(g) .
$$

Observe that from Eq. (11) one can prove both that $\chi_{g}^{\omega}\left(g_{1}^{\prime}\right) \chi_{g}^{\omega}\left(g_{2}^{\prime}\right)=\chi_{g}^{\omega}\left(g_{1}^{\prime} g_{2}^{\prime}\right)$ (i.e. $\chi_{g}^{\omega}=\varphi_{\omega}(g)$ is in $\left.G^{*}\right)$ and that $\chi_{g_{1}}^{\omega} \chi_{g_{2}}^{\omega}=\chi_{g_{1} g_{2}}^{\omega}$ (i.e. $\varphi_{\omega}$ is a homomorphism). For the particular case of a maximally non-commutative projective representation of a finite abelian group, the kernel of $\varphi_{\omega}$ [which is equal to $G(\omega)$ in general] is trivial, and therefore $\varphi_{\omega}$ is an isomorphism; that is, for any $\chi \in G^{*}$ there is a unique $g \in G$ such that $\chi_{g}^{\omega}=\chi$.

We construct the unitary $\mathcal{D}_{\omega}$ corresponding to the generalized Kennedy-Tasaki transformation (as we will see later, it maps from the maximal symmetry-breaking phase into the SPT phase), acting on a chain of $N$ sites with open boundary conditions, according to

$$
\mathcal{D}_{\omega}=\sum_{\chi} \Omega_{\omega}\left(\varphi_{\omega}^{-1}(\chi)\right)|\chi\rangle\langle\chi|,
$$

where we use the abbreviations $\chi=\left(\chi_{1}, \ldots, \chi_{N}\right)$, $\varphi_{\omega}^{-1}(\boldsymbol{\chi})=\left(\varphi_{\omega}^{-1}\left(\chi_{1}\right), \ldots, \varphi_{\omega}^{-1}\left(\chi_{N}\right)\right)$, and $\Omega_{\omega}(\mathbf{g})$ is the phase factor defined such that

$$
V_{\omega}\left(g_{N}\right) \cdots V_{\omega}\left(g_{1}\right)=\Omega_{\omega}(\mathbf{g}) V_{\omega}\left(g_{N} \cdots g_{1}\right) .
$$

(here, as in Eq. (8), the sum is over the characters $\chi$ that appear in the representation.) 
For the case of a spin-1 chain with $D_{2}$ symmetry, one can check directly that the choice of factor system $\omega$ defined by the projective representation Eq. (7) gives

$$
V\left(a_{N}\right) \cdots V\left(a_{1}\right)=(-1)^{\sum_{j<k} \mu\left(a_{j}\right) \nu\left(a_{k}\right)} V\left(a_{N} \cdots a_{1}\right),
$$

(where, loosely, one obtains a phase factor of -1 for every $V(z)=\sigma_{z}$ operator to the left of a $V(x)=\sigma_{x}$ operator). Therefore, Eq. (12) reduces to the standard KennedyTasaki transformation Eq. (3) if we choose this factor system. Note that the definition of $\mathcal{D}_{\omega}$ is not the same for different factor systems $\omega$ within the same cohomology class. However, the difference is not very significant; see Appendix A.

Due to the way the unitary $\mathcal{D}_{\omega}$ is defined, we can immediately derive the basic property that, although it is a non-local transformation, for any symmetry-respecting observable $A$ supported on a block of $n$ sites, the transformed observable $\mathcal{D} A \mathcal{D}^{\dagger}$ is still supported on the same block. We will use the notation $\chi=\left(\chi_{l}, \chi_{b}, \chi_{r}\right)$, corresponding to grouping the sites in the chain according to whether they are, respectively, to the left of, within, or to the right of the block containing the support of A. Thus the matrix element $\left\langle\chi_{l}, \chi_{b}, \chi_{r}|A| \xi_{l}, \xi_{b}, \xi_{r}\right\rangle$ can be nonzero only if $\chi_{l}=\xi_{l}, \chi_{r}=\xi_{r}$, and $\prod_{j=1}^{n} \chi_{b, j}=\prod_{j=1}^{n} \xi_{b, j}$ (the last condition comes from the assumption that $A$ commutes with the symmetry). As a result, it is easy to show from the definition of $\Omega$ [Eq. 13)] that $\Omega_{\omega}\left(\varphi_{\omega}^{-1}(\chi)\right) \Omega_{\omega}\left(\varphi_{\omega}^{-1}(\xi)\right)^{-1}=$ $\Omega_{\omega}^{(n)}\left(\varphi_{\omega}^{-1}\left(\chi_{b}\right)\right) \Omega_{\omega}^{(n)}\left(\varphi_{\omega}^{-1}\left(\xi_{b}\right)\right)^{-1}$, and hence that $\mathcal{D}_{\omega} A \mathcal{D}_{\omega}^{\dagger}=\mathcal{D}_{\omega}^{(n)} A \mathcal{D}_{\omega}^{(n) \dagger}$, where $\Omega_{\omega}^{(n)}$ and $\mathcal{D}_{\omega}^{(n)}$ are defined as $\Omega_{\omega}$ and $\mathcal{D}_{\omega}$ would be if the $n$ sites in the block constituted the entire chain.

\section{ACTION OF THE GENERALIZED KT TRANSFORMATION ON A GENERALIZED AKLT STATE}

Although we have focussed on the transformation of the Hamiltonian under the generalized KT transformation $\mathcal{D}_{\omega}$, for illustrative purposes we will consider in this section a particular Hamiltonian within the SPT phase described by cohomology class $[\omega]$, for which the ground state subspace can be found analytically. We calculate explicitly how this subspace transforms under $\mathcal{D}_{\omega}^{\dagger}$, and show that the transformed ground state subspace reflects the spontaneous breaking of the symmetry in the bulk. The definition of $\mathcal{D}_{\omega}$ [Eqs. [12) and (13)] arises naturally out of this discussion.

Affleck, Kennedy, Lieb and Tasaki (AKLT) ${ }^{19}$ constructed a system in the Haldane phase for which the ground state can be represented exactly as a "valencebond solid", or (in more modern language) a matrixproduct state (MPS) ${ }^{20}$. We will now define a generalization of the AKLT ground state for the SPT phase with cohomology class $[\omega]$. We will write it for open boundary conditions, which means we have to define a subspace $\mathcal{P}$ of states corresponding to the degenerate ground-state subspace. The states in this subspace are of the MPS form

$$
\sum_{\chi} \operatorname{Tr}\left(A_{\chi_{N}} \cdots A_{\chi_{1}} B\right)|\chi\rangle,
$$

where we set $A_{\chi}=V_{\omega}\left(\varphi_{\omega}^{-1}(\chi)\right)$ (where $V_{\omega}$ is an irreducible projective representation with factor system $\omega$ ), and the subspace comprises the states obtained from all possible $D \times D$ matrices $B$ (with $D$ the dimension of $\left.V_{\omega}\right)$. The theory of MPS parent Hamiltonians 20 allows one to construct a local frustration-free Hamiltonian for which $\mathcal{P}$ is the $D^{2}$-fold degenerate ground state subspace. From the classification of SPT order in matrix-product states 78 , one can show ${ }^{12}$ that $H$ indeed lies in the SPT phase described by cohomology class $[\omega]$.

We will only consider the state $|\Psi\rangle$ resulting from setting $B=\frac{1}{D} \sum_{g} V(g)^{\dagger}$, as it turns out that applying $[u(g)]^{\otimes N}$ to $|\Psi\rangle$ for group elements $g$ generates a basis for $\mathcal{P}$. This gives

$$
|\Psi\rangle=\sum_{\chi} \Omega_{\omega}\left(\varphi_{\omega}^{-1}(\chi)\right)|\chi\rangle,
$$

where we have used the fact that $\operatorname{Tr}\left[V_{\omega}(h) V_{\omega}(g)^{\dagger}\right]=$ $D \delta_{g, h}$ (which follows from the fact that $\operatorname{Tr} V_{\omega}(g)=0$ for $g \neq 1$, a consequence of maximal non-commutativity). This means that

$$
\mathcal{D}_{\omega}^{\dagger}|\Psi\rangle=\sum_{\chi}|\chi\rangle=|\phi\rangle^{\otimes N},
$$

where $|\phi\rangle=\sum_{\chi}|\chi\rangle$. Since $[u(g)]^{\otimes N}$ commutes with $\mathcal{D}_{\omega}^{\dagger}$, a basis for the transformed subspace $\mathcal{D}_{\omega}^{\dagger} \mathcal{P}$ comprises the states $\left\{[u(g)]^{\otimes N}|\phi\rangle^{\otimes N}, g \in G\right\}$. Thus the transformed Hamiltonian under $\mathcal{D}_{\omega}^{\dagger}$ indeed has a set of locally distinguishable symmetry-breaking ground states, as we expect.

\section{STRING ORDER}

A key property of the Kennedy-Tasaki transformation is that it relates two-particle correlations (which are expected to be long-ranged in the maximal symmetrybreaking phase for the $Z_{2} \times Z_{2}$ symmetry) to the string correlation functions that characterize the Haldane phase. Here, we will establish a similar correspondence for our general construction. This property will also allow us to determine how the generalized Kennedy-Tasaki transformation maps between different quantum phases.

\section{A. Symmetry-breaking phases and two-particle correlations}

Let us first give a general discussion of the twoparticle correlations that we expect to see in the maximal symmetry-breaking phase (i.e. where the subgroup 
of symmetry operations that are not spontaneously broken in the bulk is trivial) for an on-site abelian symmetry. A system in this phase will have a collection of degenerate symmetry-breaking ground states. Traditionally, the symmetry-breaking is detected through the nonzero value of an order parameter, which is the expectation value of a single-site observable $A$ such that $\langle A\rangle=0$ for any symmetry-respecting state. For example, in the case of the quantum transverse-field Ising model on spin- $1 / 2$ 's, with Hamiltonian

$$
H=-\sum_{i} \sigma_{i}^{z} \sigma_{i+1}^{z}+\lambda \sum_{i} \sigma_{i}^{x},
$$

the appropriate order parameter is $\left\langle\sigma_{z}\right\rangle$. The fact that $\sigma_{x} \sigma_{z} \sigma_{x}=-\sigma_{z}$ ensures that $\left\langle\sigma_{z}\right\rangle$ must be zero for any state respecting the spin-flip symmetry $\prod_{i} \sigma_{i}^{x}$. In the general case, we can consider an observable $A$ such that

$$
u\left(g^{\prime}\right) A u\left(g^{\prime}\right)^{\dagger}=\chi\left(g^{\prime}\right) A
$$

for some $\chi \in G^{*}$. By a similar argument as before, for $\chi \neq 1$ we find that $\langle A\rangle=0$ for any symmetry-respecting state. As another example, in the case of a spin-1 chain with the $D_{2}$ rotation symmetry, the spin-component operators $S_{a}(a=x, y, z)$ satisfy Eq. 19p for appropriate choices of $\chi$.

Denote the space of operators $A$ satisfying Eq. (19) by $\mathcal{A}_{\chi}$. For a given symmetry-breaking state, a given operator in $\mathcal{A}_{\chi}$ could still have zero expectation value by accident. However, we will now argue that, for a given maximal symmetry-breaking state, for every nontrivial $\chi \in G^{*}$ a generic choice of $A \in \mathcal{A}_{\chi}$ will reveal the symmetry-breaking through its nonzero expectation value. Indeed, let $\rho$ be the reduced state density operator on a single site. It suffices to show that the subspace $\mathcal{B}_{\chi}=\left\{A \in \mathcal{A}_{\chi} \mid \operatorname{Tr}(A \rho)=0\right\}$ is a proper subspace (i.e. $\mathcal{B}_{\chi} \neq \mathcal{A}_{\chi}$ ). Suppose by way of contradiction that $\mathcal{B}_{\chi}=\mathcal{A}_{\chi}$. Then every $A \in \mathcal{A}_{\chi}$ must satisfy $\operatorname{Tr}(A \rho)=0$. But since the set $\left\{\left|\chi^{\prime}\right\rangle\left\langle\chi^{\prime} \chi\right|: \chi^{\prime} \in G^{*}\right\}$ comprises a basis for $\mathcal{A}_{\chi}$, this would imply that $\left\langle\chi^{\prime} \chi|\rho| \chi^{\prime}\right\rangle=0$ for all $\chi^{\prime}$. In the maximal symmetry breaking phase, the ground state has no residual symmetry, and hence there is no constraint on the reduced state $\rho$ that would force all of these matrix elements to be zero [whereas if the ground state were invariant under the symmetry operation corresponding to the group element $g$, then this would force $\left\langle\chi_{1}|\rho| \chi_{2}\right\rangle=0$ for all $\chi_{1}, \chi_{2} \in G^{*}$ such that $\chi_{1}(g) \neq \chi_{2}(g)$.] Certainly, for a generic state in the maximal symmetry-breaking phase, these matrix elements would not all be zero.

An alternative measure of the symmetry-breaking is the two-particle correlation function $\left\langle C_{n}(A, B)\right\rangle$ (for $\left.A, B \in \mathcal{A}_{\chi}\right)$, where

$$
C_{n}(A, B) \equiv A^{\dagger} \otimes \mathbb{I}^{\otimes(n-2)} \otimes B .
$$

Because each of the symmetry-breaking ground states should be short-range correlated, the correlation function $\left\langle C_{n}(A, B)\right\rangle$ converges to $\langle A\rangle^{*}\langle B\rangle$ as $n \rightarrow \infty$. The expectations here are taken with respect to a particular choice of symmetry-breaking ground state, but notice that $C_{n}(A, B)$ commutes with the symmetry, and therefore its expectation is independent of this choice.

\section{B. SPT phases and string correlation functions}

A key feature of the Haldane phase and its generalizations is that there is no locally-detectable symmetry breaking in the bulk, and consequently all the twoparticle correlations $\left\langle C_{n}(A, B)\right\rangle$ decay exponentially as $n \rightarrow \infty$. Nevertheless, such SPT phases still have a more subtle form of long-range order detectable through string correlations. As a result, we are led to consider the following generalization of Eq. 20 (reducing to it when $g=1)$ :

$$
C_{n}(A, B ; g)=A^{\dagger} \otimes[u(g)]^{\otimes(n-2)} \otimes B .
$$

(Recall that $u(g)$ is the unitary on-site action of the symmetry.) In particular, the den Nijs-Rommelse string operators ${ }^{2}$ for the Haldane phase,

$$
S^{\alpha} \otimes\left[e^{i \pi S^{\alpha}}\right]^{\otimes(n-2)} \otimes S^{\alpha}=C_{n}\left(S^{\alpha}, S^{\alpha} ; \alpha\right), \quad \alpha=x, y, z,
$$

with $S^{\alpha}$ the spin-component operators, are of this form. In the Haldane phase, the den Nijs-Rommelse string correlations are long-ranged, i.e. $\lim _{n \rightarrow \infty}\left\langle C_{n}\left(S^{\alpha}, S^{\alpha} ; \alpha\right)\right\rangle \neq$ 0 .

Traditionally, long-ranged string correlations have been viewed as evidence of non-trivial order. However, there is a need for caution: the limiting string correlation functions $\lim _{n \rightarrow \infty}\left\langle C_{n}(A, B ; g)\right\rangle$ are nonzero for generic choices of $A$ and $B$ whenever the symmetry is unbroken in the bulk, and need not reflect any nontrivial SPT order ${ }^{1421}$. Therefore, in order to obtain useful criteria for identifying SPT phases, we must restrict ourselves to restricted classes of $A$ and $B$. Indeed, it turns out to be useful to require, as in the symmetrybreaking case, $A, B \in \mathcal{A}_{\chi}$ for some linear character $\chi$. In that case, the selection rule discussed in Ref. 14 forces $\lim _{n \rightarrow \infty}\left\langle C_{n}(A, B ; g)\right\rangle=0$ when $\varphi_{\omega}(g) \neq \chi$, where $\varphi_{\omega}$ is the homomorphism induced by the cohomology class [Eq. [10] ] (see Appendix C for the proof). In the case $\varphi_{\omega}(g)=\chi$, there is no such selection rule and so we expect that the corresponding string correlation will generically be long-ranged. (It can be checked that the latter case is the relevant one for the den Nijs-Rommelse string correlations in the Haldane phase.) Thus, the pattern of long-ranged string orders of the form considered is a useful way of identifying phases; we will make this idea more precise in Section VID.

\section{Mapping of correlation functions under the generalized Kennedy-Tasaki transformation}

We have established that the string operators $C_{n}(A, B ; g)$ are useful tools for identifying phases. There- 
fore, it makes sense to calculate how these operators transform under the generalized KT transformation $\mathcal{D}_{\omega}$, where $[\omega]$ is a maximally non-commutative cohomology class. This calculation is done in Appendix $B$, the result is (for $A, B \in \mathcal{A}_{\chi}$ )

$$
\mathcal{D}_{\omega} C_{n}(A, B ; g) \mathcal{D}_{\omega}^{\dagger}=C_{n}(\widetilde{A}, \widetilde{B} ; \widetilde{g})
$$

with

$$
\widetilde{A}=A W_{\omega}(\chi)^{\dagger}, \quad \widetilde{B}=B W_{\omega}^{\prime}(\chi)^{\dagger}, \quad \widetilde{g}=g \varphi_{\omega}^{-1}(\chi),
$$

where

$$
\begin{aligned}
& W_{\omega}(\chi)=\sum_{\chi^{\prime}} \omega\left(\varphi_{\omega}^{-1}(\chi), \varphi_{\omega}^{-1}\left(\chi^{\prime}\right)\right)\left|\chi^{\prime}\right\rangle\left\langle\chi^{\prime}\right|, \\
& W_{\omega}^{\prime}(\chi)=\sum_{\chi^{\prime}} \omega\left(\varphi_{\omega}^{-1}\left(\chi^{\prime}\right), \varphi_{\omega}^{-1}(\chi)\right)\left|\chi^{\prime}\right\rangle\left\langle\chi^{\prime}\right|,
\end{aligned}
$$

(observe that $A, B \in \mathcal{A}_{\chi}$ implies $\widetilde{A}, \widetilde{B} \in \mathcal{A}_{\chi}$ as well, since $W_{\omega}$ and $W_{\omega}^{\prime}$ commute with the symmetry).

To see the significance of this result, suppose that $[\omega]$ is a maximally non-commutative cohomology class. Recall that, in a system with maximal symmetry breaking, for any $\chi \neq 1$ we expect to be able to find $A, B \in \mathcal{A}_{\chi}$ such that $\lim _{n \rightarrow \infty}\left\langle C_{n}(A, B ; 1)\right\rangle \neq 0$. But then, by Eq. (23), this implies that in the transformed system obtained from the original one by $\mathcal{D}_{\omega}$, we will have $\lim _{n \rightarrow \infty}\left\langle C_{n}\left(\widetilde{A}, \widetilde{B} ; \varphi_{\omega}^{-1}(\chi)\right)\right\rangle \neq 0$, i.e. there are longranged string correlations of precisely the form that we expect to get in the SPT phase characterized by cohomology class $[\omega]$. In the next subsection, we will turn this into a proof that the transformed system is indeed in that phase.

\section{Patterns of string order as a "signature" for quantum phases}

We have already seen that the long-range behavior of string correlations of the form $\langle C(A, B ; g)\rangle$ (with $A, B \in$ $\mathcal{A}_{\chi}$ for some character $\chi$ ) is a useful probe for identifying different kinds of ordering in systems with a finite abelian on-site symmetry. In Appendix C, we go further, and show that this long-range behavior uniquely identifies all possible quantum phases that result from symmetry-respecting Hamiltonians. (The general classification of such phases was given in Ref. 8 and 22 , it includes conventional symmetry-breaking phases, SPT phases with no symmetry-breaking in the bulk, as well as other examples in which SPT and symmetry-breaking orders combine.)

The result of Appendix C is expressed in terms of the following "signature" function $M$ acting on $G^{*} \times G$ to measure which of the string correlations are long-ranged:

$$
M(\chi, g)= \begin{cases}1 & \text { if } \lim _{n \rightarrow \infty}\left\langle C_{n}(A, B, g)\right\rangle \neq 0 \text { generically } \\ & \text { in the phase, when } A, B \in \mathcal{A}_{\chi}, \\ 0 \quad \text { otherwise }\end{cases}
$$

We have included the word "generically", because it is possible that there might be specific points in the phase and/or choices of $A, B \in \mathcal{A}_{\chi}$ such that the limiting correlation is "accidentally" zero. [For example, in the case $g=1$, we derived in Sec. VIA the condition for a given state in the maximal symmetry-breaking phase to satisfy $\left.\lim _{n \rightarrow \infty} C_{n}(A, B ; 1)\right\rangle=0$ for all $A, B \in \mathcal{A}_{\chi}$, even though generically we expect these two-body correlations to be long-ranged.] The result of Appendix Clis then that each possible phase in the general classification has a distinct signature $M$.

Combining this result with that of the previous subsection VIC allows us to definitively establish in general how different phases are transformed into each other by the generalized KT transformation $\mathcal{D}_{\omega}$ (with $[\omega]$ a maximally non-commutative cohomology class). Indeed, suppose we start from a phase described by signature $M$. Then, by Eqs. (23) and (24), the transformed phase resulting from application of $\overline{\mathcal{D}}_{\omega}$ has signature

$$
M^{\prime}(\chi, g)=M\left(\chi, g\left[\varphi_{\omega}^{-1}(\chi)\right]^{-1}\right)
$$

In particular, we can consider the case that the starting phase is the maximal symmetry breaking phase (all the symmetries broken in the bulk). The arguments of Sec. VIA show that for such a phase, $M(\chi, 1)=1$ for all $\chi$. Furthermore, it was shown in Ref. 14 that string order of the form $\left\langle C_{n}(A, B ; g)\right\rangle$ can be long-ranged only when the symmetry corresponding to $g$ is unbroken in the bulk. Thus, the maximal symmetry-breaking phase has signature $M(\chi, g)=1 \Leftrightarrow g=1$. It follows that the transformed phase resulting from applying $\mathcal{D}_{\omega}$ has signature $M^{\prime}(\chi, g)=1 \Leftrightarrow g=\varphi_{\omega}^{-1}(\chi)$. From the discussion of Appendix C, we see that this is precisely the signature of the SPT phase with cohomology class $[\omega]$, as expected.

Note that, although in this paper we have concentrated on the duality between pure SPT order and maximal symmetry-breaking order, Eq. (28) can be used to determine in general how the generalized KT transformation relates $\mathcal{D}_{\omega}$ different symmetric phases to each other, including combined symmetry-breaking/SPT phases. For example, see Ref $\frac{15]}{10}$ for a discussion of the $Z_{N} \times Z_{N}$ case. (In this case, our generalized KT transformation reduces to the one defined in Ref. ${ }^{[15}$, or a variant thereof, depending on which cohomology class $[\omega]$ and factor system representative $\omega$ one uses in the construction).

\section{THE KENNEDY-TASAKI TRANSFORMATION FOR CONTINUOUS SYMMETRIES}

We stress that our assumption of a maximallynoncommutative cohomology class of a finite abelian group might not be as restrictive as it sounds. Indeed, an SPT phase characterized by an arbitrary group could still be identified as part of a maximally non-commutative SPT phase with respect to a finite abelian subgroup. As 
an example, here we will discuss how our framework allows us to apply the concept of hidden symmetry breaking to some generalizations of the Haldane phase.

Just as the Haldane phase is motivated by $\mathrm{SO}(3)$ invariant antiferromagnets, these generalized Haldane phases contain systems that are invariant under an $\mathrm{SO}(2 k+1)$ or $\mathrm{SU}(k)$ symmetry. However, in each case, we will identify a finite abelian subgroup (analogous to $D_{2}$ for the Haldane phase), which will turn out to be the relevant one for identifying the hidden symmetry breaking. In each case, this finite abelian subgroup will turn out to be sufficient to classify the phases, since imposing the full continuous symmetry does not separate any phases that could not already be distinguished through this subgroup. This suggests that, even when the full continuous symmetry is present, we should describe the SPT order in terms of the hidden breaking of the finite abelian subgroup.

\section{A. The $\mathrm{SO}(2 k+1)$ Haldane phase}

For systems invariant under an on-site $\mathrm{SO}(2 k+1)$ symmetry, there is exactly one non-trivial SPT phase 11123 , which we can think of as a generalization of the Haldane phase (reducing to it in the case $k=1$ ). The corresponding cohomology class is that of the spinor representations of $\mathrm{SO}(2 k+1)$ (which are, in fact, projective representations).

Identifying $\mathrm{SO}(2 k+1)$ with its representation in terms of $(2 k+1) \times(2 k+1)$ orthogonal matrices with unit determinant, we define $G_{k} \equiv\{A \in \mathrm{SO}(2 k+1)$ : $A$ is diagonal in the standard basis $\}$, which constitutes a finite abelian subgroup. We can construct a minimal set of generators $\left\{u^{(l)}, l=1, \ldots, 2 k\right\}$ with matrix elements

$$
u_{i, j}^{(l)}=(-1)^{1-\delta_{i, l}} \delta_{i, j}
$$

(we do not include $u^{(2 k+1)}$ in our of minimal set of generators because it is not independent of the rest; indeed, $\left.u^{(2 k+1)}=\prod_{l=1}^{2 k} u^{(l)}\right)$. This shows that $G_{k} \cong Z_{2}^{\times 2 k}$. It can be shown, by considering the restriction of the spinor representations of $\mathrm{SO}(2 k+1)$ to the subgroup $G_{k}$, that the cohomology class of $G_{k}$ for systems in the non-trivial SPT phase with respect to $\mathrm{SO}(2 k+1)$ is that of the projective representation generated by

$$
V\left(u^{(l)}\right)=\Gamma_{l},
$$

where the $2 k$ matrices $\Gamma_{l}$ obey the anti-commutation relations $\left\{\Gamma_{a}, \Gamma_{b}\right\}=2 \delta_{a, b}$. It is straightforward to show that this cohomology class is maximally non-commutative.

Thus, we can use our general prescription [Eq. [12] ] to construct a generalized KT transformation for systems in the non-trivial SPT phase with respect to $\mathrm{SO}(2 k+1)$. In analogy to the original KT transformation for the Haldane phase (which breaks the full rotation symmetry, preserving only the discrete subgroup $D_{2}$ ), the resulting transformed system will only have the discrete $Z_{2}^{\times 2 k}$ symmetry instead of the full $\mathrm{SO}(2 k+1)$. Furthermore, the transformed system will be in a maximal local symmetrybreaking phase for this discrete symmetry. In this sense, the non-trivial $\mathrm{SO}(2 k+1)$ SPT phase can be understood as a result of the "hidden breaking" of the $Z_{2}^{\times 2 k}$ symmetry. Note that, if we make a particular choice of factor system within the appropriate cohomology class, it can be shown that the generalized Kennedy-Tasaki transformation constructed according to our general prescription [see Eq. [12] ] coincides with the one constructed in Ref. [11.

We remark that, since for the $\mathrm{SO}(2 k+1)$ symmetry group there is only one nontrivial SPT phase, it can already be distinguished from the trivial phase via a $Z_{2} \times Z_{2}$ subgroup. Although one could therefore construct the generalized KT transformation $\mathcal{D}_{\omega}$ based on the $Z_{2} \times Z_{2}$ subgroup, as in Ref. 15, we prefer to construct it based on $Z_{2}^{\times 2 k}$. This ensures that the transformed phase is maximally symmetry-breaking. If one instead uses only the $Z_{2} \times Z_{2}$ subgroup to construct $\mathcal{D}_{\omega}$, it can be shown (using similar arguments to Sec. VID and Appendices B and C) that the resulting phase breaks $Z_{2} \times Z_{2}$, but is still SPT-ordered with respect to the remaining $Z_{2}^{\times 2(k-1)}$.

In general, a useful way to ensure that the transformed phase has no residual SPT order is by counting the ground state degeneracy: if the degeneracy of the original SPT phase is fully explained by the symmetrybreaking in the transformed phase, then there cannot be any residual SPT order. For instance, consider the $\mathrm{SO}(2 k+1)$ case. We can assume that the fractionalized representation of the symmetry on the edge is the fundamental spinor representation, as it suffices to confirm the lack of residual SPT order at a single point in the phase. There is then a $2^{k}$-fold degeneracy associated with each edge, which agrees with the $2^{2 k}$-fold degeneracy we expect for a phase that maximally breaks a $Z_{2}^{\times 2 k}$ symmetry. A similar property also holds for the $\mathrm{SU}(k)$ example considered in the next section.

\section{B. SPT phases for $\mathrm{SU}(k)$}

Instead of thinking of the Haldane phase as invariant under an $\mathrm{SO}(3)$ symmetry, we can also think of it as invariant under $\mathrm{PSU}(2) \equiv \mathrm{SU}(2) /\{+1,-1\}$. Of course, $\mathrm{PSU}(2) \cong \mathrm{SO}(3)$, but this suggests an alternative generalization of the Haldane phase: one that is invariant under an on-site representation of $\operatorname{PSU}(k) \equiv \mathrm{SU}(k) / C_{k}$, where $C_{k}=\{\exp (2 i \pi l / k): l=0, \ldots, k-1\}$.

Given the definition of $\operatorname{PSU}(k)$, we can construct a finite abelian subgroup by identifying a subgroup of $\mathrm{SU}(k)$ that is abelian up to phase factors (i.e. up to elements of $C_{k}$ ). The discrete Heisenberg-Weyl group is such a subgroup; it is the group generated [in the standard rep- 
resentation of $\mathrm{SU}(k)]$ by the two operators

$$
\begin{aligned}
& X=\frac{1}{W} \sum_{l=0}^{k-1}|(l+1) \bmod k\rangle\langle l|, \\
& Z=\frac{1}{W} \sum_{l=0}^{k-1} w^{l}|l\rangle\langle l|
\end{aligned}
$$

where $w$ is a primitive $k$-th root of unity, and we have included the normalization factor $W=w^{(k-1) / 2}$ to ensure that $\operatorname{det} X=\operatorname{det} Z=1$. The fact that the subgroup generated by $X$ and $Z$ is abelian up to phases follows from the relation $Z X=w X Z$. The abelian subgroup of $\operatorname{PSU}(k)$ corresponding to the Heisenberg-Weyl group is isomorphic to $Z_{k} \times Z_{k}$. The cohomology group for $Z_{k} \times Z_{k}$ is $H^{2}\left(Z_{k} \times Z_{k}, \mathrm{U}(1)\right) \cong Z_{k} \cong H^{2}(\mathrm{PSU}(k), \mathrm{U}(1))$, and it can be shown ${ }^{[15}$ that the $k$ cohomology classes of $\mathrm{PSU}(k)$ correspond exactly to the $k$ cohomology classes of the $Z_{k} \times Z_{k}$ subgroup. Thus, the $Z_{k} \times Z_{k}$ subgroup is sufficient to characterize all the SPT phases even in the presence of the full $\operatorname{PSU}(k)$ symmetry. In order to apply our generalized Kennedy-Tasaki transformation, we need a maximally non-commutative cohomology class; if we let $\left[\omega_{0}\right]$ be a generator for the cohomology group $H^{2}\left(Z_{k} \times Z_{k}, \mathrm{U}(1)\right)$, then it can be shown that $\left[\omega_{0}^{l}\right]$ is maximally non-commutative if and only if $l$ and $k$ are coprime.

\section{Other continuous symmetry groups}

We leave it as an open question whether a similar analysis to the above for $S O(2 k+1)$ and $S U(k)$ holds for other continuous symmetry groups. In Ref.[15, it is shown that a subgroup of the form $Z_{N} \times Z_{N}$ can be found for all the cases involving classical Lie groups. However, as we have shown with the $S O(2 k+1)$ example in Sec. VII A, this is not the whole story, especially if the aim is to identify a relevant generalised KT transformation that can 'fully' remove the SPT order, i.e., that can relate a SPT-ordered phase to a maximally symmetry-breaking phase.

\section{THE TOPOLOGICAL DISENTANGLER}

In this section, we will briefly outline a physical interpretation of the resulting correspondence between the SPT ground states and the symmetry-breaking ground states, in terms of the entanglement structure of the ground states.

When we group sites together in blocks of size $\gg \xi$, with $\xi$ the correlation length, any gapped ground state starts to resemble (up to on-site unitary rotations on the blocked sites) a "dimer state" which can be viewed as a renormalization fixed point ${ }^{24}$. If the ground state is in an SPT phase characterized by a maximally noncommutative cohomology class, this dimer state will take the form shown in Fig. 1(a); this is a consequence of
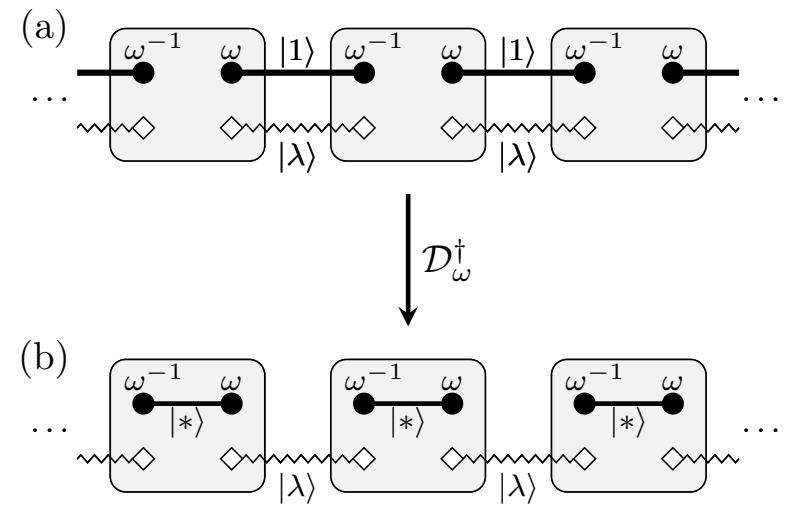

FIG. 1. (a) The "dimer state" renormalization fixed point for the SPT phase corresponding to a maximally commutative cohomology class $[\omega]$; (b) The result of applying $\mathcal{D}_{\omega}^{\dagger}$, for a particular choice of boundary conditions. Each shaded area represents one coarse-grained site. The black dots transform under irreducible projective representations with factor systems $\omega$ and $\omega^{-1}$ under the symmetry, and the diamonds do not transform at all under the symmetry. Note: Two adjacent black dots transform linearly under the symmetry; therefore, we can introduce the simultaneous eigenbasis $\{|\chi\rangle\}$ of the symmetry (with the states labelled by linear characters $\chi$; from Schur's Lemma it follows that they must be maximally entangled). For $\chi=1$ this gives the state $|1\rangle$ appearing in (a). In (b), we have defined $|*\rangle=\sum_{\chi}|\chi\rangle$. The state $|\lambda\rangle$ is not universal and depends on the specific point in the phase.

the fact that a maximally non-commutative factor system corresponds to a unique irreducible projective representation 17118 . We see that the entanglement between two halves of the chain has two origins: the universal "topological" entanglement represented by the maximally entangled state $|1\rangle$, and the "non-topological" entanglement represented by the state $|\lambda\rangle$. (The "dot" and "diamond" particles correspond to the "protected" and "junk" subsystems discussed in Ref. 12,)

It is therefore instructive to consider what happens under $\mathcal{D}_{\omega}^{\dagger}$ to a ground state of the form shown in Fig. 1(a). The calculation required is similar to that of Sec. V. (Indeed, when written in a matrix-product state form, the state of Fig. 1(a) reduces to Eq. (15) when the "extra" particles in the $|\lambda\rangle$ state are absent.) Here we just state the result: for a suitable choice of boundary conditions, the resulting state is as depicted in Fig. 11(b); note that this resulting state is no longer invariant under the symmetry, and the orbit of this state under the symmetry is the set of symmetry-breaking ground states for the transformed system. We see that the topological component of the entanglement has been eliminated, with the nontopological part of the state remaining untouched. In this sense, we can think of the generalized KT transformation $\mathcal{D}_{\omega}^{\dagger}$ as a "topological disentangler" 25 . 


\section{DISCUSSION}

We have presented a generalization of the KennedyTasaki transformation, which maps certain onedimensional models with SPT order to ones with traditional symmetry-breaking. This formulation further expands the characterization of SPT order as a form of hidden symmetry breaking to a broad class of models, specifically those for which the SPT order can be related to a maximally non-commutative factor system of a finite abelian group that acts on the system through an on-site unitary representation. Whether any analogous results hold in higher dimensions, or for other kinds of symmetries (e.g. time reversal), remains an open question. We point out, however, that in two dimensions and higher there is a different kind of duality that holds for any onsite unitary representation of a finite group $G$ : between SPT phases and topological lattice gauge theories with gauge group $G^{2627}$.

We have also interpreted the action of our generalized KT transformation as a topological disentangler 25 , removing the topological component of the entanglement from the ground state. Transformations that remove entanglement from a quantum many-body system have found use in numerical methods such as the multiscale entanglement renormalisation ansatz (MERA) 2 , and so may the generalized KT transformation presented here.

\section{ACKNOWLEDGMENTS}

We acknowledge support from the ARC via the Centre of Excellence in Engineered Quantum Systems (EQuS), project number CE110001013.

\section{Appendix A: Choice of representative factor system}

Here we will discuss the difference between $\mathcal{D}_{\omega}$ and $\mathcal{D}_{\omega^{\prime}}$, where

$$
\omega^{\prime}(g, h)=\beta(g) \beta(h) \beta(g h)^{-1} \omega(g, h),
$$

[for some set of phase factors $\beta(g)$ ] is another factor system in the same cohomology class as $\omega$. The important thing to consider is the transformed Hamiltonians resulting from the respective transformations. Thus we will only consider the way $\mathcal{D}_{\omega}$ acts on symmetry-respecting observables $A$. First of all we observe that the isomorphism $\varphi_{\omega}$ only depends on the cohomology class, so that $\varphi_{\omega}=\varphi_{\omega^{\prime}}$. It is then straightforward to show that

$$
\mathcal{D}_{\omega^{\prime}} A \mathcal{D}_{\omega^{\prime}}^{\dagger}=b_{\beta}^{\otimes N} \mathcal{D}_{\omega} A \mathcal{D}_{\omega}^{\dagger} b_{\beta}^{\otimes N \dagger}
$$

where we have defined

$$
b_{\beta}=\sum_{\chi} \beta\left(\varphi_{\omega}^{-1}(\chi)\right)|\chi\rangle\langle\chi| .
$$

Therefore, the two transformed Hamiltonians differ only be a rephasing of the basis on each site. Clearly, this does not change the nature of the resulting transformed phase.

\section{Appendix B: Proof of Eq. (23)}

For simplicity of notation, we use the isomorphism $\varphi_{\omega}$ to label our site basis by group elements instead of group characters, i.e. we define

$$
|g\rangle \equiv\left|\varphi_{\omega}(g)\right\rangle
$$

For some choice of character $\chi_{*}$, let $A, B \in \mathcal{A}_{\chi_{*}}$, and define $g_{*}=\varphi_{\omega}^{-1}\left(\chi_{*}\right)$. This implies that

$$
\begin{aligned}
A^{\dagger} & =\sum_{g} \mu_{g}|g\rangle\left\langle g g_{*}\right| \\
B & =\sum_{g} \nu_{g}\left|g g_{*}\right\rangle\langle g| .
\end{aligned}
$$

for some scalars $\left\{\mu_{g}\right\}$ and $\left\{\nu_{g}\right\}$. Thus,

$$
A^{\dagger} \otimes \mathbb{I}^{\otimes(n-2)} \otimes B=\sum_{g_{1}, \ldots, g_{n}} \mu_{g_{1}} \nu_{g_{n}}\left|g_{1}, g_{2}, \ldots, g_{n} g_{*}\right\rangle\left\langle g_{1} g_{*}, \ldots, g_{n-1}, g_{n}\right|
$$

Therefore

$$
\mathcal{D}_{\omega}\left(A^{\dagger} \otimes \mathbb{I}^{\otimes(n-2)} \otimes B\right) \mathcal{D}_{\omega}^{\dagger}=\sum_{g_{1}, \ldots, g_{n}} \mu_{g_{1}} \nu_{g_{n}} \Gamma_{g_{1}, \ldots, g_{n}}\left|g_{1}, g_{2}, \ldots, g_{n} g_{*}\right\rangle\left\langle g_{1} g_{*}, \ldots, g_{n-1}, g_{n}\right|
$$

where $\Gamma_{g_{1}, \ldots, g_{n}}$ is the phase factor such that

$$
V_{\omega}\left(g_{n} g_{*}\right) V_{\omega}\left(g_{n-1}\right) \cdots V_{\omega}\left(g_{1}\right)=\Gamma_{g_{1}, \ldots, g_{n}} V_{\omega}\left(g_{n}\right) \cdots V_{\omega}\left(g_{2}\right) V_{\omega}\left(g_{1} g_{*}\right)
$$


This gives

$$
\Gamma_{g_{1}, \ldots, g_{n}}=\omega\left(g_{n}, g_{*}\right)^{-1} \alpha_{\omega}\left(g_{*}, g_{n-1}\right) \cdots \alpha_{\omega}\left(g_{*}, g_{2}\right) \omega\left(g_{*}, g_{1}\right),
$$

where $\alpha_{\omega}(g, h)$ is the phase factor such that

$$
V_{\omega}(g) V_{\omega}(h)=\alpha_{\omega}(g, h) V_{\omega}(h) V_{\omega}(g)
$$

However, now comparing with Eq. 11, we find that

$$
u(g)|h\rangle=\alpha_{\omega}(g, h)|h\rangle
$$

Therefore, we can conclude that

$$
\mathcal{D}_{\omega}\left(A^{\dagger} \otimes \mathbb{I}^{\otimes(n-2)} \otimes B\right) \mathcal{D}_{\omega}^{\dagger}=\left[W_{\omega}\left(g_{*}\right) A^{\dagger}\right] \otimes\left[u\left(g_{*}\right)\right]^{\otimes(n-2)} \otimes\left[B W_{\omega}^{\prime}\left(g_{*}\right)^{\dagger}\right],
$$

which leads to Eq. 23, where we have defined

$$
W_{\omega}\left(g_{*}\right)=\sum_{g} \omega\left(g_{*}, g\right)|g\rangle\left\langle g\left|, \quad W_{\omega}^{\prime}\left(g_{*}\right)=\sum_{g} \omega\left(g, g_{*}\right)\right| g\right\rangle\langle g|
$$

\section{Appendix C: Identifying phases from patterns of string order}

Symmetry-breaking phases and SPT phases are two different kinds of phases that can arise in one-dimensional systems invariant under an on-site symmetry. As was shown in Ref. 8 and 22 , the most general kind of phase for such systems combines both aspects. A general symmetric phase for a symmetry group $G$ is characterized by a subgroup $H$ (corresponding to the symmetries that are unbroken in the bulk) and a cohomology class $[\omega]$ for $H$, such that each of the degenerate symmetry-breaking ground states is in the SPT phase $[\omega]$ with respect to the subsymmetry. In this Appendix, we will show that, in the case of finite abelian symmetry groups $G$, each distinct phase gives rise to a distinct pattern of long-range string correlations, as defined through the "signature" function $M$ of Eq. 27.

Let us first consider the case of pure SPT phases (i.e. $H=G$, and the phases are classified by cohomology classes of $G$ ). It was argued in Ref. 21 that, if the operators $A$ and $B$ are chosen at random, then generically one finds that $\left\langle C_{n}(A, B ; g)\right\rangle \neq 0$ is nonzero for any ground state that is invariant under the symmetry in the bulk (which will be the case for any pure SPT phase as well as the trivial phase). Suppose, however, that we instead choose $A, B \in \mathcal{A}_{\chi}$ for some character $\chi$. Then, whenever $\chi \neq \varphi_{\omega}(g)$, we must be able to find some $g^{\prime} \in G$ such that $\chi\left(g^{\prime}\right) \neq\left[\varphi_{\omega}(g)\right]\left(g^{\prime}\right)$. Recalling the definition of $\varphi_{\omega}$ [Eq. [10], and of $\mathcal{A}_{\chi}$ [Section VIA], this implies that $\alpha_{1} \neq \alpha_{2}$, where $\alpha_{1}, \alpha_{2}$ are the scalars such that

$$
\begin{aligned}
V_{\omega}(g) V_{\omega}\left(g^{\prime}\right) & =\alpha_{1} V_{\omega}\left(g^{\prime}\right) V_{\omega}(g), \\
A u\left(g^{\prime}\right) & =\alpha_{2} u\left(g^{\prime}\right) A
\end{aligned}
$$

As shown in Ref. 14, there is a selection rule that prevents the string correlation $\left\langle C_{n}(A, B ; g)\right\rangle$ from be- ing long-ranged when $\alpha_{1} \neq \alpha_{2}$. On the other hand, if $\chi=\varphi_{\omega}(g)$ then there is no such selection rule and we expect that $\left\langle C_{n}(A, B ; g)\right\rangle$ will generically be longranged even with the constraint $A, B \in \mathcal{A}_{\chi}$. In summary, therefore, the pure SPT phase has the signature $M(\chi, g)=1 \Leftrightarrow \chi=\varphi_{\omega}(g)$. For example, if the cohomology class is maximally non-commutative, then $\varphi_{\omega}$ is invertible and thus, for each character $\chi$, there is a unique $g$ such that $M(\chi, g)=1$, and vice versa. At the other extreme, if the cohomology class is trivial, then $\varphi_{\omega}(g)=1$ for all $g$, and thus $M(\chi, g)=1 \Leftrightarrow \chi=1$.

The above arguments show that the possible signature functions $M$ are in one-to-one correspondence with the homomorphisms $\varphi_{\omega}$. In order to establish that distinct SPT phases correspond to different signature functions, it only remains to show that if $\omega_{1}$ and $\omega_{2}$ have different cohomology classes, then $\varphi_{\omega_{1}} \neq \varphi_{\omega_{2}}$. Since $\varphi_{\omega}$ is linear in $\omega$, it suffices to prove that if $\varphi_{\omega}=1$ (the trivial homomorphism), then $\omega$ has trivial cohomology class. Indeed, $\varphi_{\omega}=1$ implies, by definition of $\varphi_{\omega}$, that $V_{\omega}(g) V_{\omega}\left(g^{\prime}\right) V_{\omega}(g)^{\dagger}=V_{\omega}\left(g^{\prime}\right)$ for all $g, g^{\prime} \in G$, which is to say all the elements $V_{\omega}(g)$ commute. If we choose $V_{\omega}$ to be irreducible, then Schur's Lemma implies that $V_{\omega}(g)=\beta(g)$ for some scalar phase factors $\beta(g)$. Therefore, the projective representation $V_{\omega}$ has trivial cohomology class.

Now let us return to the general case, where phases are classified by a subgroup $H \leq G$ and a cohomology class [ $\omega]$ of $H$. Because all the order parameters we are considering are expectation values of symmetry-respecting operators, we just need to determine their value for a single symmetry-breaking ground state. Since each of these symmetry-breaking ground states lies in an SPT phase with respect to the subsymmetry $H$, we find that, for $h \in H, M(\chi, h)=1$ if and only if $\chi_{H}=\varphi_{\omega}(h)$, where $\chi_{H}$ is the restriction $\chi$ onto the subgroup $H$. On the other 
hand, if $g \notin H$ then $M(\chi, g)=0$ for any $\chi$ (because the symmetry operation corresponding to $h$ is broken in the bulk $\left.{ }^{21}\right)$. In summary, the signature of a general symmetric phase is $M(\chi, g)=1 \Leftrightarrow\left[g \in H\right.$ and $\left.\chi_{H}=\varphi_{\omega}(g)\right]$. Notice that, for $H \neq G$, there will exist non-trivial characters $\chi$ such that $\chi_{H}=1$, which implies that $M(\chi, 1)=1$. Recall that this corresponds to nonzero values of

$$
\lim _{n \rightarrow \infty}\left\langle A^{\dagger} \otimes \mathbb{I}^{\otimes n} \otimes B\right\rangle
$$

(for $A, B \in \mathcal{A}_{\chi}$ ), which is what we expect, since for $H \neq$ $G$ there is partial symmetry-breaking, and therefore there should also be long-range order.

We will now prove that no two symmetric phases can have the same signature. Arguing as in the pure SPT case, it is easy to see that for a fixed $H$ all distinct phases have different signatures. To complete the proof, we will now show that the subgroup $H$ can be recovered from the signature, and therefore two phases with different $H$ must have different signatures. To do this we make use of the following result:
Lemma 1. Let $G$ be a finite abelian group, and let $H$ be a subgroup. Then any linear character acting on $H$ can be extended to a linear character on $G$. That is, for any $\xi \in H^{*}$, there exists $\chi \in G^{*}$ such that $\chi_{H}=\xi$.

Proof. Define the homomorphism $\psi: G^{*} \rightarrow H^{*}, \chi \mapsto$ $\chi_{H}$. Observe that $\operatorname{ker} \psi \cong(G / H)^{*}$, and therefore $|\operatorname{ker} \psi|=\left|(G / H)^{*}\right|=|G / H|=|G| /|H|$. But $\psi\left(G^{*}\right) \cong$ $G^{*} / \operatorname{ker} \psi$, so $\left|\psi\left(G^{*}\right)\right|=\left|G^{*}\right| /|\operatorname{ker} \psi|=|G| /|\operatorname{ker} \psi|=$ $|H|=\left|H^{*}\right|$. It follows that $\psi\left(G^{*}\right)=H^{*}$, i.e. $\psi$ is surjective.

Lemma 1 ensures that, for any $h \in H$, we can find a character $\chi \in G^{*}$ such that $\chi_{H}=\varphi_{\omega}(h)$, and hence $M(\chi, h)=1$. By contrast, if $g \notin H$ then we found above that $M(\chi, g)=0$ for all $\chi \in G^{*}$. Therefore, the subgroup $H$ can be recovered from the signature according to

$$
H=\left\{h \in G \mid M(\chi, h)=1 \text { for some } \chi \in G^{*}\right\} .
$$

This completes the proof that distinct phases have distinct signatures.
1 Xie Chen, Zheng-Cheng Gu, and Xiao-Gang Wen, "Local unitary transformation, long-range quantum entanglement, wave function renormalization, and topological order," Phys. Rev. B, 82, 155138 (2010), arXiv:1004.3835.

2 Marcel den Nijs and Koos Rommelse, "Preroughening transitions in crystal surfaces and valence-bond phases in quantum spin chains," Phys. Rev. B, 40, 4709 (1989)

3 E. Polizzi, F. Mila, and E. S. Sørensen, " $S=1 / 2$ chain-boundary excitations in the Haldane phase of onedimensional $S=1$ systems," Phys. Rev. B, 58, 2407 (1998)

${ }^{4}$ Frank Pollmann, Ari M. Turner, Erez Berg, and Masaki Oshikawa, "Entanglement spectrum of a topological phase in one dimension," Phys. Rev. B, 81, 064439 (2010), arXiv:0910.1811

5 F. Verstraete, M. A. Martín-Delgado, and J. I. Cirac, "Diverging entanglement length in gapped quantum spin systems," Phys. Rev. Lett., 92, 087201 (2004), arXiv:0311087.

${ }^{6}$ L. Campos Venuti and M. Roncaglia, "Analytic relations between localizable entanglement and string correlations in spin systems," Phys. Rev. Lett., 94, 207207 (2005) arXiv:cond-mat/0503021.

7 Xie Chen, Zheng-Cheng Gu, and Xiao-Gang Wen, "Classification of gapped symmetric phases in onedimensional spin systems," Phys. Rev. B, 83, 035107 (2011) arXiv:1008.3745

$\checkmark$ Norbert Schuch, David Pérez-García, and Ignacio Cirac, "Classifying quantum phases using matrix product states and projected entangled pair states," Phys. Rev. B, 84, 165139 (2011), arXiv:1010.3732

y Tom Kennedy and Hal Tasaki, "Hidden $Z_{2} \times Z_{2}$ symmetry breaking in Haldane-gap antiferromagnets," Phys. Rev. B, 45, 304-307 (1992) "Hidden symmetry breaking and the Haldane phase in $S=1$ quantum spin chains," Comm. Math. Phys., 147, 431 (1992)

${ }^{10}$ Frank Pollmann, Erez Berg, Ari M. Turner, and Masaki
Oshikawa, "Symmetry protection of topological phases in one-dimensional quantum spin systems," Phys. Rev. B, 85, 075125 (2012), arXiv:0909.4059

${ }^{11}$ Hong-Hao Tu, Guang-Ming Zhang, and Tao Xiang, "String order and hidden topological symmetry in the $\mathrm{SO}(2 n+1)$ symmetric matrix product states," J. Phys. A: Math. Theor., 41, 415201 (2008), arXiv:0804.1685.

12 Dominic V. Else, Ilai Schwarz, Stephen D. Bartlett, and Andrew C. Doherty, "Symmetry-protected phases for measurement-based quantum computation," Phys. Rev. Lett., 108, 240505 (2012), arXiv:1201.4877.

is Dominic V. Else, Stephen D. Bartlett, and Andrew C. Doherty, "Symmetry protection of measurement-based quantum computation in ground states," New J. Phys., 14, 113016 (2012), arXiv:1207.4805

14 Frank Pollmann and Ari M. Turner, "Detection of symmetry-protected topological phases in one dimension," Phys. Rev. B, 86, 125441 (2012), arXiv:1204.0704

15 Kasper Duivenvoorden and Thomas Quella, "From symmetry-protected topological order to landau order," (2013), arXiv:1304.7234

16 M Oshikawa, "Hidden $Z_{2} \times Z_{2}$ symmetry in quantum spin chains with arbitrary integer spin," J. Phys.: Condens. Matter, 4, 7469 (1992)

if Ya. G. Berkovich and E. M. Zhmud', Characters of Finite Groups, Vol. 1 (American Mathematical Society, Providence, Rhode Island, 1998).

18 R. Frucht, "Über die darstellung endlicher abelscher gruppen durch kollineationen," J. Reine Angew. Math., 1932, $16(1932)$

${ }^{19}$ I. Affleck, T. Kennedy, E.H. Lieb, and H. Tasaki, "Valence bond ground states in isotropic quantum antiferromagnets," Comm. Math. Phys., 115, 477 (1988) Ian Affleck, Tom Kennedy, Elliott H. Lieb, and Hal Tasaki, "Rigorous results on valence-bond ground states in antiferromagnets," Phys. Rev. Lett., 59, 799 (1987) 
20 D. Perez-Garcia, F. Verstraete, M. M. Wolf, and J. I. Cirac, "Matrix product state representations," Quant. Inf. Comput., 7, 401 (2007), arXiv:quant-ph/0608197.

21 D. Pérez-García, M. M. Wolf, M. Sanz, F. Verstraete, and J. I. Cirac, "String order and symmetries in quantum spin lattices," Phys. Rev. Lett., 100, 167202 (2008) arXiv:0802.0447

22 Xie Chen, Zheng-Cheng Gu, and Xiao-Gang Wen, "Complete classification of one-dimensional gapped quantum phases in interacting spin systems," Phys. Rev. B, 84, 235128 (2011), arXiv:1103.3323

23 Kasper Duivenvoorden and Thomas Quella, "On topological phases of spin chains," Phys. Rev. B, 87, 125145 (2013) arXiv:1206.2462

${ }^{24}$ F. Verstraete, J. I. Cirac, J. I. Latorre, E. Rico, and M. M. Wolf, "Renormalization-group transformations on quantum states," Phys. Rev. Lett., 94, 140601 (2005) arXiv:quant-ph/0410227.

25 Kouichi Okunishi, "Topological disentangler for the valence-bond-solid chain," Phys. Rev. B, 83, 104411 (2011), arXiv:1011.3277.

20 Michael Levin and Zheng-Cheng Gu, "Braiding statistics approach to symmetry-protected topological phases," Phys. Rev. B, 86, 115109 (2012), arXiv:1202.3120

27 Ling-Yang Hung and Xiao-Gang Wen, "Quantized topological terms in weakly coupled gauge theories and their connection to symmetry protected topological phases," (2012), arXiv:1211.2767

${ }^{28}$ G. Vidal, "Entanglement renormalization," Phys. Rev. Lett., 99, 220405 (2007), arXiv:cond-mat/0512165

29 G. Vidal, "Class of quantum many-body states that can be efficiently simulated," Phys. Rev. Lett., 101, 110501 (2008), arXiv:quant-ph/0610099. 